\title{
Marcadores discursivos en el habla de la ciudad de Cartagena de Indias ${ }^{*}$
}

\author{
Liliam Cuartas López ${ }^{1}$ \\ Universidad de Cartagena
}

\section{Resumen}

La sociolingüística estudia una o varias lenguas en su contexto social y proporciona fundamentos para explicar, de manera más adecuada, cómo las características sociales de los sujetos determinan la variación en el uso del discurso. Ofrece, así mismo, las herramientas metodológicas suficientes para desarrollar un riguroso y confiable estudio sobre la lengua hablada. En este sentido, nuestra investigación tiene como objetivo determinar, desde un enfoque sociolingüístico y discursivo, la función de los "marcadores discursivos" (según los tipos de discurso: narrativo, descriptivo, argumentativo o expositivo) en el habla de la ciudad de Cartagena de Indias. El estudio evidenciará que estos cumplen diferentes funciones en el discurso y que algunas variables sociales independientes son determinantes en la frecuencia de uso de los marcadores, así como en ciertas secuencias discursivas.

\section{Abstract}

Sociolinguistics is the field of study of one or more languages in their social context and provides a foundation to more appropriately explain how the social characteristics of individuals determine the variation in speech use. It also offers sufficient methodological tools to develop a rigorous and reliable analysis of spoken language. From this sociolinguistic perspective, our research aims to determine the role of "discourse markers" (according to the types of discourse: narrative, descriptive, argumentative or expository) in the speech of Cartagena de Indias, Colombia. This study will prove that these markers serve different discourse functions, and that some independent social variables are crucial to the frequency of the markers and certain discourse sequences.
Palabras clave: sociolingüística, marcadores discursivos, lengua, habla, variables sociales.
Key words: sociolinguistics, discourse markers, language, speech, social variables.

\footnotetext{
* Discourse markers in the speech of Cartagena de Indias, Colombia. Recibido: Marzo, 2011 - Aprobado: Julio, 2011.

${ }^{1}$ Licenciada en Españoly Literatura de la Universidad de Córdoba. Magíster en Lingüística de la Universidad Nacional. Docente en las e-mail: liliamcuartas@hotmail.com
} 


\section{Introducción}

Los trabajos que relacionan lengua y sociedad empiezan a cobrar relevancia a partir de los años sesenta. El vínculo entre ambas fue abordado de manera diferente por investigadores de diversos campos $^{2}$. La sociolingüística, como una de esas disciplinas, no sólo estudia la lengua (o varias lenguas) en su contexto social, sino que, además, proporciona fundamentos para explicar mejor cómo las características sociales de los sujetos pueden determinar la variación en el uso del discurso. Así mismo, ofrece las herramientas metodológicas suficientes para desarrollar un riguroso y confiable estudio sobre la lengua hablada.

Gracias a su carácter interdisciplinar, la sociolingüística ha propiciado grandes avances en los estudios del lenguaje, abordando diversos temas desde todos los niveles de la lengua. Estos adelantos se han dado tanto en el ámbito internacional como en el ámbito nacional. No obstante, los estudios realizados aún no dan cuenta de la variedad lingüística existente en un país pluricultural, multilingüe y multidialectal como Colombia. Es en este sentido que, para la última década, en el Caribe colombiano han surgido con fuerza investigaciones sobre la variedad dialectal regional. Tal es el caso del Proyecto para el Estudio Sociolingüístico del Español de España y América en Barranquilla (PRESEEA-Barranquilla, 2002) ${ }^{3}$ que

${ }^{2}$ Trudgill y Hernández Campoy (2007) afirman que, atendiendo a los objetivos, es posible dividir los estudios de lenguaje y sociedad en tres grupos: i) objetivos sociológicos: aquellos estudios en los que los objetivos son completamente sociológicos o científico-sociales, y por lo tanto no pertenecen a la sociolingüística, como la etnometodología; ii) objetivos sociológicos y lingüísticos: aquellos estudios en los que los objetivos son en parte sociológicos y en parte lingüísticos: donde entrarían la sociología del lenguaje, la psicología social del lenguaje, la lingüística antropológica, el análisis del discurso, la etnografía del habla o los estudios del lenguaje y género; y iii) objetivos lingüísticos: aquellos estudios en los que los objetivos son puramente lingüísticos, como la dialectología tradicional, la lingüística secular, la geolingüística, la sociolingüística histórica y la sociolingüística criolla. De este modo se diferencian aquellos estudios sociolingüísticos por naturaleza de aquellos que claramente no lo son, dado que utilizan datos lingüísticos sólo con fines sociológicos.

${ }^{3}$ El Proyecto para el Estudio Sociolingüístico del Español de España y América (PRESEEA) surgió con la finalidad de recoger un corpus sociolingüístico del Español de las principales ciudades del mundo hispánico. Su planteamiento se hizo por primera vez en al marco del X Congreso de la Asociación de Lingüística y Filología de la América Latina (ALFAL, 1993) y se constituye finalmente en el XI Congreso (1996). Desde entonces muchos investigadores independientes y otros agrupados han venido vinculándose al Proyecto, y hoy hay un gran número de trabajos en España y en América. En el 2002, el Grupo de Investigación para el Estudio Sociolingüístico 
describe sociolingüísticamentela comunidad de habla barranquillera, aunque admite que se desconoce la situación sociolingüística de los ocho núcleos urbanos principales del Caribe colombiano hispánico.

En Cartagena, por ejemplo, pese a su importancia histórica, cultural, económica, turística y lingüística, se reconocen sólo dos investigaciones sociolingüísticas: Fonología de las consonantes implosivas en el español urbano de Cartagena de Indias (Colombia), de Becerra (1985), y Dynamic synchrony in the Spanish of Cartagena, Colombia, de Lafford (1982, citada por Silva-Corvalán, 2001). En este marco surge esta investigación, cuyo fin es determinar, desde un enfoque sociolingüístico y discursivo, la función de los marcadores según los tipos de discurso (narrativo, descriptivo, argumentativo y expositivo) en el habla de la ciudad de Cartagena de Indias. Ello implica recoger un corpus sociolingüístico de la ciudad, el cual permita desarrollar estudios de orden lingüístico, social y cultural; caracterizar los marcadores discursivos de los hablantes cartageneros según la secuencia discursiva; determinar la función de los marcadores usados por los cartageneros, y establecer la covariación entre el uso de los marcadores discursivos y los factores sociales como sexo, edad, o nivel de instrucción.

Portolés (2001) define los marcadores discursivos como:

[...] unidades lingüísticas invariables que no ejercen una función sintáctica en el marco de la predicación oracional y poseen un cometido coincidente en el discurso: el de guiar, de acuerdo con sus distintas propiedades morfosintácticas, semánticas y pragmáticas, las inferencias que se realizan en la comunicación (25).

De acuerdo con lo anterior, un análisis de estas unidades exige recurrir a disciplinas como la pragmática y el análisis del discurso, para dar una explicación de la funcionalidad e intención de dichas partículas en los actos comunicativos. Los aportes que ha hecho la teoría del discurso, la gramática y la teoría de la comunicación permiten revelar las funciones que adquieren estas unidades en secuencias discursivas como la descriptiva, expositiva, narrativa y argumentativa.

del Español en el Caribe Colombiano Hipánico (GIESCA) emprendió la ardua tarea de recolectar el Corpus de las principales ciudades del Caribe colombiano, tarea que aún no termina; vinculándose así al Proyecto PRESEEA internacional, desde donde se denomina al desarrollado por GIESCA como PRESEEA-Barranquilla (como se le referenciará de ahora en adelante). 
En el contexto nacional, en cuanto a marcadores discursivos, los aportes son escasos. El Caribe colombiano es, tal vez, donde se han realizado los primeros trabajos sobre esta variable sociolingüística en Colombia. Estos estudios son: "Marcadores discursivos en el habla de Barranquilla" (Rodríguez Cadena, 1999), y a partir del corpus de PRESEEA-Barraquilla, el estudio monográfico: "Los marcadores discursivos en la comunidad de habla de Barranquilla" (Vásquez Cantillo, 2005) y "Tendencia de uso de los marcadores discursivos en el habla de Valledupar" (Calderón Noguera, 2006).

Así pues, la presente investigación contribuye no sólo con la recolección del corpus sociolingüístico de la ciudad de Cartagena, como complemento del macroproyecto para el Estudio Sociolingüístico de Español de España y América en el Caribe hispánico (PRESEEABarranquilla, 2002), sino que, además, aporta elementos teóricos de basealmarco investigativoy académicodelos estudios sociolingüísticos que vienen adelantándose sobre marcadores discursivos.

La recolección del corpus, que implica una caracterización social de la ciudad de Cartagena de Indias, permitirá realizar investigaciones sociolingüísticas de variables fónicas, morfosintácticas, léxicosemánticas y discursivas, que permitan explicar la variación lingüística en esta comunidad de habla, comparables con otras que se hayan realizado con corpus de la misma naturaleza en otras ciudades de Hispanoamérica y España.

\section{Análisis cualitativo de los marcadores discursivos}

Este capítulo presenta un análisis cualitativo de las secuencias discursivas que se hallan en las entrevistas y de las funciones de los diferentes marcadores discursivos con una clasificación de los mismos. Luego, da cuenta de un análisis cuantitativo de los marcadores por cada una de las variables sociales: edad, sexo/ género y nivel de instrucción; y de los marcadores de las secuencias discursivas: narrativa, descriptiva, argumentativa, explicativa y dialogal, por las variables sociales mencionadas.

\subsection{Análisis cualitativo}

\subsubsection{Las secuencias discursivas}

En las intervenciones de los entrevistados se identificaron diversas secuencias textuales (o discursivas) que pueden aparecer con frecuencia en conversaciones cotidianas como consecuencia del tipo de pregunta, de los temas de conversación y de la agilidad del 
entrevistador. Las tipologías discursivas halladas son: narrativa, descriptiva, argumentativa, expositivas y dialogal. Los lingüistas del texto señalan que generalmente se combinan más de una secuencia en un texto, aunque sobresale una de ellas.

En el corpus de Cartagena, algunos temas facilitan la utilización de ciertas secuencias. Por ejemplo, es común que al hablar de política y religión prevalezcan secuencias argumentativas. Cuando se conversa sobre la ciudad (pasado), el barrio (pasado) o la familia, es común que se recurra a las estructuras narrativas y también, con frecuencia, a las descriptivas. Las explicativas predominan en temas que exigen dar instrucciones o que muestran una secuencia u orden, como las actividades diarias, recetas de comida, entre otros. Ahora bien, no se puede afirmar que haya una relación causa-consecuencia entre temas y secuencias discursivas.

Los marcadores discursivos son uno de los elementos que caracterizan las secuencias discursivas por cuanto contribuyen en su estructuración. De ahí que sea necesario evidenciar y caracterizar, en principio, algunas muestras halladas de éstas en las conversaciones semidirigidas del corpus de Cartagena de Indias, para posteriormente analizar la función y la frecuencia de los marcadores en dichas secuencias. En la siguiente intervención predomina la secuencia narrativa.

S: porque ya le digo, en en Bocagrande, lo que había era el pueblo ese, no había más nada como en la actualidad, entonces de ahí ahí nació la familia mía de parte de madre y la familia mía de parte de padre, nacieron en San Antero. Entonces la vieja mía se unió con él, se casaron y nació la primera hermana mía que se llamaba Juana xxxxxx aquí en el barrio Getsemaní y en Bocagrande, los que estuvieron los que nacieron allá fue ehh Esther y Alicia, de ahí salimos, nos llevó pa`San Antero pande es su tierra natal de mi papá, ya en San Antero allá nació el hermano mayor mío Catalino y de xxxxxx Santos que soy yo (SDD, HN1G3) ${ }^{4}$

\footnotetext{
${ }^{4}$ Las primeras letras son las iniciales del nombre del informante. Después de la coma, encontramos una letra que indica el sexo del informante (" $\mathrm{H}$ ", hombre o "M", mujer). La letra y el número que sigue alude al nivel de instrucción ("N", nivel y "1" Primaria), y la letra el número final, la generación (“G”, generación y "3" primera $<55+$ años $>$ ) Asimismo, se utilizan las "xxx" para reservar el nombre del hablante. Por lo demás, La transcripción de las entrevista, para este caso, es de forma literal, como hablan los informantes. Por ende, en estos ejemplos elido el etiquetado.
} 
En el texto anterior el informante cuenta cómo se conformó su familia, cómo fue ganando integrantes hasta obtener los miembros actuales. Así, en el texto se observa la transformación de predicados a través de un proceso (principio, desarrollo, fin). Es decir, los episodios contados se presentan en un orden cronológico. Los tiempos también contribuyen a mostrar el proceso, la transformación y el cambio, a establecer ese orden cronológico: primero nacieron los padres; después se unieron, se casaron; nació la primera hermana, estuvieron en San Antero hasta el nacimiento del último hijo, el cual, es quien cuenta la historia. Y esto reafirma que los tiempos preferidos en las estructuras narrativas son el pretérito perfecto simple y el imperfecto de indicativo (para las acciones secundarias): "había", "era", "llamaba", "nació", "nacieron", "se unió", "se casaron", "y nació", "salimos", "nos llevó", y sustantivos temporales como "la actualidad".

También se puede observar que la función representativa es la que predomina en el texto. En este caso, el narrador cuenta cómo fue la conformación de su núcleo familiar. Se sabe ahora que después de que sus padres se casaron nacieron en el orden respectivo: Juana, Esther, Alicia, Catalino y Santos (el que narra). El hablante cuenta algo que está fuera de sí mismo, pertenece a un mundo externo, objetivo. De ahí que se sitúe como narrador de una serie de hechos que, generalmente, localiza en su pasado y expresa cómo van cambiando. Esta secuencia muestra, adicionalmente, elementos temporales, los deícticos: "ahí nació", "familia mía", "la vieja mía", "hermana mía", "aquí en el barrio", "nacieron allá", "de ahí salimos", "allá nació". Por otra parte, las secuencias expositivas son propias de las definiciones y caracterizaciones.

A: pero el guía en su perfil profesional, yo he podido notar ambas diferencias ahora que estoy haciendo ese ese trabajo de campo porque existen tres generaciones de guías: la primera que fue de empíricos, la segunda que fue de guías con formación que se encargó de formarlos la Academia de Historia y el Sena. Ya en la década del sesenta y ya en la tercera generación aparezco yo como actor anónimo, inicialmente porque yo no soy guía. Yo estudié, inicialmente, fue Administración de Empresas Turísticas y cuando llego aquí al Sena me encuentro con que estoy vinculado a un programa de hotelería y turismo; y hay un programa de guías y me toca dictar los cursos que tienen que ver con guías. Entonces desde el año noventa y dos, empiezo a formar guías, pero sin esa conciencia ni con esa ehh competencia tal, sino desde la academia, desde la experiencia universitaria 
que obtenía el conocimiento y se lo trasmitía a ellos lo que tiene que ver con el turismo. Después sí viene la exigencia de conocer la ciudad y recorrerla y aparecen las nuevas generaciones de guías, la tercera y la cuarta, que ya no son guías solamente con formación en el Sena sino que necesitan conocer idiomas y algunos se matriculan en el Colombo, aprenden inglés; otros en la Alianza aprenden francés, entonces hay un guía empírico que nace que ni siquiera estudió pero que tiene una vocación clara de que la profesión lo beneficia. Hay un guía con formación en el Sena y aparece la tercera generación, que son los guías que además del Sena se dan cuenta que a Cartagena llega turismo internacional y que necesitan aprender otros idiomas (AM, HN3G2).

V: hay hemano usté uno coge y compra su sábalo, lo arregla con su su limón, lo frita y se le echa todo su bastimento, lo último que se va hacer que se va echar es el sábalo, el pecado eso y se le echa una miguita de zumo de coco y eso queda aunque yo no lo como a mí el pecado no me guta (VMQ, MN1G3)

Laintervención del informante hombre (AM, HN3G2) es una secuencia expositiva en la que reflexiona sobre un tema en particular. En este caso él, ahora en el ejercicio de guía turístico, ha podido "notar tres generaciones" de los que practican este oficio, que son: la "primera", la de los "empíricos"; la segunda, los que tienen "formación" por la Academia de Historia y el Sena, y la "tercera" con formación en el Sena, pero aparentemente más profunda. Sin embargo, menciona una "cuarta", que sería aquella que, adicionalmente, es bilingüe. Pero al final, cuando resume las ideas, reafirma que son tres, y agrupa las dos últimas en una misma "generación". En esta secuencia también se muestran los lazos de causalidad que ligan los hechos entre ellos, por ejemplo: "la tercera generación, que son los guías que además del Sena se dan cuenta que a Cartagena llega turismo internacional y que necesitan aprender otros idiomas".

Ya que la secuencia expositiva se caracteriza por la objetividad, las formas lingüísticas suelen ser, en el verbo, las del nivel actual (o mundo comentado) y presente: "he podido, "estoy haciendo, "existen", "aparezco", "soy guía", "llego", "me encuentro", "estoy vinculado", "hay", "me toca dictar", "tienen que ver", etc. En el texto se ofrece una caracterización de las diferentes "generaciones" de guías, lo que hace obligatoria la presencia de sustantivos y adjetivos, más que verbos de acción. La intervención de la informante (VMQ MN1G3), por su 
parte, explica cómo se prepara un Sábalo. Básicamente, es un texto instructivo, o dominantemente instruccional, como lo son las recetas de cocina. La lista de ingredientes se va presentando inmediatamente con el procedimiento, la manera y el orden en que se deben utilizar: el pescado, el limón, etc.

Esta estructura tiene como característica la presencia del imperativo (aunque en otras recetas se usa la primera persona del singular), secuencias de acciones y fórmulas apelativas: "coge", "compra", "arregla", "frita", "echa", "va a hacer", "va a echar", "echa", etc. Todos estos verbos tienen un valor apelativo claro. La lingüística del texto señala también que la forma general de progresión en los de carácter expositivo es de tema constante o de hipertema: "existen tres generaciones de guías" (AM, HN3G2) y predominan los conectores aditivos: "y", "que", "entonces", "después" (AM, HN3G2) o " $y$ ", "lo último" (VMQ MN1G3).

Resumiendo, los lingüistas del texto señalan que los discursos expositivos se caracterizan por dar información sobre datos o ideas. El lenguaje que utilizan es preciso y convincente, y se tiene especial cuidado en la selección de numerosos sustantivos y adjetivos, por cuanto transmiten mucha información. Estas secuencias se estructurarán según las necesidades de información, partiendo, por lo general, de lo conocido, para ir añadiendo datos nuevos. También tienen como rasgo primordial la objetividad. La descriptiva, por su parte, es un tipo de secuencia en la que predomina la descripción de un estado (situación, ideas, paisaje), con una visión estática.

J: Bueno el Daniel Lemetri es un barrio que al igual que casi todo en Cartagena las fiesta son cada día, cada fin de semana, no hacen fiesta de bando y cosas cuando son el 11 de noviembre aprovechan los barrios vecino, y si es un barrio generalmente tranquilo, los fines de semana siempre ponen música pero y y venden bebidas alcohólicas, pero en realidad es un barrio tranquilo desafortunadamente hay barrios alrededor, que terminan dañando el ambiente del barrio pero en sí Daniel Lemetri es un barrio tranquilo. (JM, HN2G2).

V: Bueno, cuando mi mamá compró esto, esto era una loma y esto estaba solitario nada ma había la casa de aquí de mano derecha y má y lo solares que y una casita acá atrás, el resto era puro monte, pasaba un burro vendiendo este pan con el señor Lucho que como a la tres de la tarde ese era lo único que pasaba por aquí y 
la gente que venía de allá arriba venía por el caminito porque todo era monte y uno no corría peligro, la tiendas quedaban a tres y cuatro cuadra uno tenía que ir a comprar allá abajo donde el señor Eladio Fuente y regresaba para coger el bus era en la harina Tres Catillo o por a acá donde ante, que ante estaba el teatro Miriam, que ya eso, ya eso lo abolieron, esa eran la partes ma cercanas para coger el bus nosotros y si llovía salíamos con un par de zapatos aquí y no lo poníamos otro allá adelante. (VMQ MN1G3).

CLF: añoro mi casa y que mi casa es como las casa antigua ahorita vamos pa que veas que yo tengo patio, tengo un columpio dónde me siento a leer, tengo mis matas, allí llegan los pájaros; entonces porque tengo una jaula pero no tengo pájaro, sino que yo a veces compro alpiste y le riego alpiste, tengo una hicotea, llega la maría mulata, la tortolita, la monjita y me pongo entonces a ver los pájaros que llegan, me encanta, ahora mismo hay mangos a veces lo mamones estoy pendiente del palo de anón que que ya tiene dos anones chiquitos o sea a mí me gusta la vida tranquila, yo soy de vida tranquila. (CLF, MN3G3).

En las tres intervenciones se describe la realidad. El informante JM (HN2G2) describe el ambiente del barrio Daniel Lemaitre, principalmente durante las fiestas del 11 de noviembre; VMQ (MN1G3) muestra cómo era, desde el punto de vista de su configuración física, el barrio para el que se mudó muchos años atrás, y CLF (MN3G3) describe el patio de su casa. Como se observa, no hay una organización cronológica, sino propiedades, características y relación con la situación. Por eso puede caber lo emotivo como ocurre en las descripciones anteriores, y que se refleja por los siguientes términos: "barrio tranquilo", "desafortunadamente hay barrios alrededor", "terminan dañando el ambiente, "estaba solitario, "casita acá atrá", "el resto era puro monte", "caminito, "añoro", "ahorita vamos", "la tortolita, "la monjita, "anones chiquitos", etc. Como se sabe, los adverbios, los diminutivos y algunas construcciones sintagmáticas contribuyen a este rasgo emotivo en el discurso, pero no tienen intención estética, ni buscan reelaborar el mensaje.

Se produce una correferencia y una relación hiperonímica de todoparte, que se suele expresar en el léxico: "el barrio":, "fin de semana", "fiestas de bando y cosas", "generalmente tranquilo", " música”, "bebidas alcohólicas" (JM, HN2G2); "el barrio": "una loma”, "solitario”, “casa de 
aquî", "casita acá trá", "puro monte", etc. (VMQ MN1G3); y el "patio": "columpio", "mis matas", "los pájaros", "una jaula", "alpiste", "mangos", "los mamones", "palo de anón" (CLF, MN3G3). Se diseccionan y caracterizan las partes. De ahí esa cohesión léxica evidente y la obligatoriedad de la nutrida presencia de sustantivos y adjetivos, como se observa en los listados anteriores.

Además del predominio de la adjetivación, de la acumulación de sustantivos o estructuras nominales y el no predominio de las acciones, los verbos aparecen en presente, y algunas veces en imperfecto: "es", "son", “hacen", "aprovechan”, "ponen”, “venden”, "hay", "terminan" (JM, HN2G2); "era", "estaba", "había”, pasaba”, "venía", "corría”, “tenía” (VMQ, MN1G3); "añoro", “vamos", "veas”, "tengo”, “me siento", "compro", "riego", "llega(n)", "me pongo", "hay", "estoy", "tiene”, "me gusta", "soy" (CLF, MN3G3).

El texto argumentativo siempre presenta unas razones para defender una premisa o tesis, puesto que busca salvaguardar una verdad. Véanse las intervenciones siguientes:

A: con el cambio de la constitución se legitima la con la constitución del noventa y uno se legitima la libertad de cultos; pero de hecho yo siempre he pensado que las sectas protestantes o las religiones hermanas de la católica ehh profesan una misma fe, pero la practican de forma diferente; entonces las prácticas religiosas a veces por el desconocimiento de la doctrina ehh hacen creer a quienes profesan la fe cristiana o a quienes profesamos la fe cristiana que estamos siguiendo caminos distintos, estamos es siguiendo un mismo camino pero de diferentes prácticas; es como el conocimiento que es universal sin embargo, lo importante es reconocer que uno nace en un contexto histórico y que ese contexto histórico le da cierta información cultural que le permite tener unas creencias y esas creencias se le convierten en valores y se le convierten en filosofía de vida; entonces yo nací en familia católica y soy católico y profeso la religión católica y sin embargo, comparto con muchos amigos de aquí del Sena y con mis alumnos comparto cualquier tipo de religión por mi formación filosófica. (AM, HN3G2).

CL: Claro, claro claro y entonces el mismo pensamiento de los padres de familia que como no tienen formación ellos no le conceden el valor que tiene el conocimiento; 
entonces no le importa que los niños aprendan o no les interesan es que ganen el año; por eso es que yo siempre he dicho que la peor infamia que pudo hace el gobierno de Colombia fue la promoción automática porque yo fui maestra antes de la promoción automática y después de la promoción automática; antes de la promoción automática los padres de familia se esmeraban pero ya después nada les interesaba, era que ganaran el año, ya no compraban cuaderno, aunque yo les decía traigan así sea un cuaderno viejo y aquí le engrapamos las hojas viejas pero nada, no llevaban nada, no le ayudaban, a mi una vez una mamá en el mes de marzo había un niño, yo tenía segundo grado, había un niño que no conocía ni la 0, entonces cuando yo me di cuenta de eso yo mandé a buscar la mamá y le dije mira aquí tenemos una dificultad pero que el niño de segundo debía saber leer no leer a la perfección pero sí por lo menos a desglosar, entonces vamos hacer una cosa vamos a ponernos las pilas tu y yo con él, ay, no seño pero déjelo que pierda el año, oye pero como me vas a decir a mí en el mes de marzo que déjalo que pierda el año si ni tú has hecho lo que tienes que hace ni él ha hecho lo que tiene que hacer ni yo he hecho lo que tengo que hacer como me vas a decir eso pero eso que lectura es no les importa el conocimiento; una vez llevaron un niñito de preescolar prendío en fiebre bajo un aguacero y la maestra de preescolar le ha dicho al papá que lo llevó, el papá con botas y todo eso, cuando fue a bajar el niño hay si el niño tiene fiebre como me lo traes con este aguacero y con fiebre, ¿usted sabe lo que jode ese pelao en la casa? (CLF, MN3G3).

En la primera, el informante AM (HN3G2) trata de sustentar la idea de que: "las sectas protestantes o las religiones hermanas de la católica ehh profesan una misma fe pero la practican de forma diferente"; mientras que la informante CLF (MN3G3) sostiene que "el mismo pensamiento de los padres de familia que como no tienen formación ellos no le conceden el valor que tiene el conocimiento entonces no le importa que los niños aprendan o no, les interesan es que ganen el año [...]" o "[...] que la peor infamia que pudo hace el gobierno de Colombia fue la promoción automática [...]".

En los textos argumentativos anteriores los informantes hacen uso de la exposición en algunos apartes, estrategia de la que se valen para la presentación de la tesis: "[...] con la constitución del noventa y 
uno se legitima la libertad de culto[...]", pero también para presentar una contradicción, o hacer una demostración de puntos de vistas disímiles: "[...] como el conocimiento que es universal, sin embargo, lo importante es reconocer que uno nace en un contexto histórico y que ese contexto histórico le da cierta información cultural que le permite tener unas creencias[...]”, “[...] saber leer no leer a la perfección pero sí por lo menos a desglosar[...]", y finalmente, la justificación razonada de los argumentos expuestos.

Con el discurso argumentativo, los hablantes buscan persuadir, convencer, defender una idea. Por eso es indispensable que las proposiciones tengan un desarrollo lógico y una organización adecuada en el discurso, para que exista coherencia y cohesión que garanticen, en parte, ese propósito. Eso se puede ver en ambas intervenciones, donde se evidencia una clara estructuración de las ideas que facilita que el interlocutor entienda y pueda adherirse a sus tesis. "todos, en el fondo, profesamos la misma religión" y "hoy día a los padres les interesa más que sus hijos ganen el año que lo que puedan aprender, todo lo cual es resultado de la promoción automática".

Se deja ver en estas proposiciones las posiciones de los hablantes, que resultan creíbles; se hace una aclaración u objeción de las ideas contrarias, y por último, un refuerzo de los argumentos planteados: "[...] las prácticas religiosas a veces por el desconocimiento de la doctrina ehh hacen creer a quienes profesan la fe cristiana o a quienes profesamos la fe cristiana que estamos siguiendo caminos distintos, estamos es siguiendo un mismo camino pero de diferentes prácticas" (AM, HN3G2); "[...] a mí una vez una mamá en el mes de marzo había un niño [...] que no conocía ni la 0 , entonces cuando yo me di cuenta de eso yo mandé a buscar la mamá y le dije mira aquí tenemos una dificultad pero que el niño de segundo debía saber leer, no leer a la perfección pero sí por lo menos a desglosar, entonces vamos hacer una cosa vamos a ponernos las pilas tú y yo con él, hay no seño pero déjelo que pierda el año [...]" (CLF, MN3G3).

Las intervenciones anteriores permiten corroborar que la dimensión argumentativa se muestra lingüísticamente en el vocabulario axiológico, las modalidades, los operadores y los conectores argumentativos. Entre estos últimos sobresalen: "pero", "entonces", "sin embargo", "porque", "por eso es que" y "aunque". En estos segmentos podría verse una cierta dimensión argumentativa, por la presencia de la evaluación intensificada: "[...] las sectas protestantes o las religiones hermanas de la católica [...]", “[...] esas creencias se le convierten en valores y se le convierten en filosofía de vida, que puede entenderse como orientada a convencer al lector [...]", “[...] traigan 
así sea un cuaderno viejo [...]”, “[...] pero nada no llevaban nada no le ayudaban"

Estos textos argumentativos, finalmente, se caracterizan por organizarse de modo tal que se exponen unas razones (argumentos) que llevan a unas determinadas conclusiones. Buscan influir al oyente en sus ideas, no en sus acciones. Este paso se da a través de un soporte, ley de paso, garante, topos, o inferencia.

La secuencia dialogal tiene al intercambio como la unidad constituyente. Lo que se pretende con esta estructura discursiva es entablar una conversación con el interlocutor del tipo pregunta-respuesta o cualquier otro par adyacente.
E: (risas)
C: entonces pienso yo que lo mejor sería invertirlo
E: ya
C: invertirlo
E: bueno viejo Cesar, la verdad es que para mi nuevamente un placerazo porque
C: (risas)
C: no profesor tranquilo, usted sabe a la orden
E: es eses una cuestión interesante en la medida en que esa información que nos está sirviendo para lo que estamos haciendo
C: claro claro
E: y que hayas tenido esa disponibilidad de de
C: no, no, no
E: de proponerlo dos veces me parece magnifico
C: no, no, no usted sabe
E: la verdad te agradezco muchísimo. (CA, HN3G1).

_no, ya esas cosas ya viejas ya eso bótalas.

_nuevos hijos, nuevo esposo, nuevo todo.

Ya las cosas serían diferentes ya, serían otros hijos (tos mía), otro compañero, o sea otro modo ya de vivir diferente.

_eh, bueno, Marcelina, le agradezco su colaboración.

bueno, pues mu(palabra cortada), un gracias a usted por haberme estesea, cómo se dice? (interrupción complementaria:_entrevistado), entrevistado, en este programa que usted me ayudado para hablar de las cosa pasada, de lo presente, del futuro.

_recordar ¿no? 
_recordar mi niñé y ahora que doy una persona, persona ya grande, que ya tengo mis hijos grandes tengo nietos, me siento bien gracias a Dios.

_eh(muletilla alargada), ¿Señora Marcelina nos recuerda su nombre completo?

_ MPM

_ Bueno, señora Marcelina, muchas gracias y que tenga un buen día.

_ Bueno pue, se te agradezca, doy laj gracia a usted por bejme, sea recordado cosaj vieja y cosaj nueva.

_bueno, gracias usted. (MPM, MN1G2).

Los intercambios anteriores se aparecen para facilitar la transición en el desarrollo temático: "C: invertirlo/ E: bueno viejo Cesar la verdad es que para mí nuevamente un placerazo porque/C: (risas)/C: no profesor tranquilo, usted sabe a la orden [...]". Se destacan en ellos la función fática o de contacto, para mantener abierto el canal por medio de la interacción permanente de los interlocutores: "E: $y a$;" "C: claro claro"; "_recordar ¿no?”. En cuanto a la estructura secuencial, la conversación tiene secuencias que le son propias: las de inicio y fin, que son puramente interactivas, fruto de la especial situación comunicativa que reflejan. Dichas cadenas están ritualizadas: los saludos y las despedidas. Véanse, por ejemplo: "E: bueno viejo Cesar la verdad es que para mi nuevamente un placerazo porque/C: (risas)/

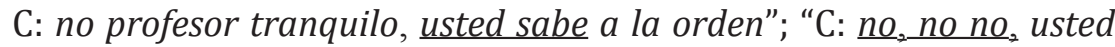
sabe/ E: la verdad te agradezco muchísimo” (CA, HN3G1); “_Bueno, señora Marcelina, muchas gracias y que tenga un buen día./_ Bueno pue, se te agradezca, doy laj gracia a usted por bejme, sea recordado cosaj vieja y cosaj nueva./_bueno, gracias usted" (MPM, MN1G2). Estas fórmulas, además de agradecer la información ofrecida, también son una manera de finalizar la conversación y despedirse.

Los lingüistas del texto afirman que los textos dialogados son especiales, en cuanto los agentes se van intercambiando. Esta situación interactiva específica es lo que exige unidades igualmente específicas. Así, se ha visto la intervención y el intercambio como unidades intermedias entre el enunciado y el texto. De las anteriores secuencias analizadas, algunas son más predominantes en las entrevistas; por ejemplo, la narrativa, pues con frecuencia los hablantes cuentan anécdotas que se estructuran con este tipo de secuencias. Cabe resaltar que la secuencia descriptiva es la menos predominante, fundamentalmente en los hombres, ya que las mujeres describen en mayor medida. Aunque esta secuencia, en comparación con la frecuencia de aparición de las otras, sigue siendo la menos utilizada en sus conversaciones. Sin olvidar que 
la secuencia dialogal engloba a las demás, debido a que el formato del texto es una entrevista. Por ello, resulta sumamente importante el desarrollo de esta secuencia discursiva en la que se aprecian las otras modalidades.

Ahora bien, como se señalaba anteriormente, la mayor aparición de una u otra secuencia puede estar condicionada por los temas abordados, por las intenciones desatadas en los enunciados y las diferentes fuerzas en la enunciación. Más adelante se podrá apreciar en términos cuantitativos las secuencias discursivas más recurrentes en las entrevistas.

\subsubsection{La función de los marcadores discursivos}

En la comunidad de habla de Cartagena se encontraron diferentes tipos de marcadores, entre ellos se hallaron los: "reformuladores explicativos", "marcadores apelativos que intentan verificar la información", "marcadores de cierre del discurso", "marcadores de concreción", "marcadores de relleno", "marcadores veredictivos", "marcadores evaluativos", "conectores consecutivos", "marcadores de apertura", "marcadores de continuidad", "marcadores discursivos apelativo-afectivos", "marcadores discursivos referenciales con carácter indefinido", "reafirmativo modal", "marcadores contra argumentativo", "marcadores de relación jerárquica bidireccional argumentativos", "marcadores interactivos", en general, de "clasificación transversal", "empáticos", de "adhesión", "marcadores asentivos". Brevemente mostraré los casos más característicos.

\subsubsection{Marcadores reformuladores explicativos: "de pronto", "osea", "pues".}

Analizando el fragmento 1, se observa que el informante (CAL, HN3G1), a través del marcador "de pronto", expresa y manifiesta claramente una reconceptualización del discurso anterior, con el fin de que el interlocutor interprete de forma precisa el enunciado. Este tipo de marcadores aclara la estructura discursiva, al punto de que la información emitida en el primer enunciado proferido puede resultar más explícita al oyente. Así puede notarse en lo que sigue.

A: ¿Cómo ve la organización política de Cartagena en términos administrativos y en términos ehh organizacionales urbanos etc.? 
B: bueno ehh, yo ahorita le comentaba que yo he visto de pronto un cierto cambio ahora que tenemos la alcaldesa, ehh de pronto por el hecho de ser mujer, de pronto yo siento que de pronto está más empapada con la parte social, ah que quiere mmm meterle de pronto el hombro a la parte de educación, por medio de la secretaría de educación se han hecho algunas cosas; entonces, en la parte o sea organizacional por ley pues es lo normal no que hayan concejales que haya una gobernación que haya una alcaldía que de pronto hay que organizar la parte administrativa ya como como de pronto como que le puedo comentar descentralizar un poco que de prontoooo, esa gente los secretarios de despacho se trasladen a los a las a las a las partes más digamos más difíciles mas vulnerables para que se empapen de la situación que hay realmente sería lo ideal no que de pronto ehh cualquiera que tenga que hacer alguna diligencia no tenga que ir hasta el centro sino que de pronto por ejemplo lo que han hecho por ley las alcaldías menores. (CAL, HN3G1).

Asimismo, en el ejemplo 2 analizamos que el entrevistador emite la pregunta y el entrevistado profiere la respuesta en el primer enunciado, caracterizando el tema de la conversación, pero asume que no ha sido lo suficientemente ilustrativo, y en consecuencia, decide acudir al marcador discursivo "o sea" para expresar seguidamente, en otros términos, la caracterización que está haciendo. De este modo, da a conocer una nueva información a partir de la primera expresión, mostrando claramente su intención enunciativa, haciéndose entender de forma amplia.

2)

A: Loma del Marión. ¿Cómo describe este lu, este el ambiente por aquí?

B: Bueno el ambiente por aquí es un ambiente bueno_ sea un ambiente sano para comparado con otras partes. (JRR, H N2G1).

3)

A: ¿y del beisbol que es lo que más recuerda una anécdota importante?

B: cuando estuve que jugué yo varias pues yo jugué juego de las estrellas y también gané un partido de 20 a 0 que no me espero no me pudieron anotar un hit nadie piso base. (JRR, HN2G1). 
A: ¿Percance con?

B: Otro percance así e pequeño no ahora pasaron fueron treinta seis a, treinta y tres años pues el año pasao fue que me corté y me cogieron seis puntos fue lo único que me han cogió [...]. (JRR, HN2G1).

Como se puede notar en los casos 3 y 4 , se cumplen las mismas características anteriormente expuestas. Hay que tener en cuenta que los marcadores "de pronto" y "pues", aunque no se encuentran en la clasificación propuesta por Portolés (2001), están adscritos en la comunidad de habla cartagenera como "marcadores reformuladores explicativos", cumpliendo las mismas funciones de los otros pertenecientes a esta categoría.

\subsubsection{Marcador apelativo que intenta verificar la información: “ $¿ y a$ ?”}

En los siguientes textos orales se puede notar cómo el informante, por medio del marcador discursivo " ¿ya?" intenta captar o atrapar la atención de su oyente. Asimismo, sirve para comprobar o confirmar si el interlocutor se mantiene solícito a lo comunicado. En el texto 5 el hablante, después de enunciar de manera amplia su idea, antes de terminar lo expresado, trata de verificar lo expuesto.

\section{5)}

A: ¿me puede relatar cómo fue su infancia?

B: bueno mi infancia fue como dicen dura, porque me tocó trabajar mucho con mi papá, porque desafortunadamente no teníamos como la plata suficiente para mantenernos, entonces tuve una infancia muy dura, como dicen, trabajaba mucho con mi papá, nos íbamos pa el monte a cortar la leña y vendíamos leña, entonces, fue una infancia muy dura como le dije, tenía que pasar trabajando todos los días cuando estaba de descanso, que no iba al colegio, era muy poco el tiempo que tenía para jugar como cualquier otro niño ¿ya?, eso es lo que yo quiero que no tengan que pasar los hijos míos, que él juegue todo lo que juegue, porque uno cuando esta pequeño necesita jugar. (JLP,HN1G1).

6)

A: ¿de ajo?

B: Agüita de ajo, agüita de ajo pero quedan bien elegante, no me quedan enchumpaos sino secos iya? Bien elegante. (JRR, HN2G1). 


\subsubsection{Marcadores de Cierre del Discurso: "Bueno, ajá"}

Dichos marcadores en los enunciados 7 y 8 clausuran el discurso.

\section{7)}

A: ¿La satisfacción de la profesión?

B: 0 sea mi sas me siento realizado bien porque me gusta cuando yo le hago un trabajo a una persona a un odontólogo lo que es remodelación de equipo que consiste en pinta el equipo tapízaselo cámbiale toda la mangueras valvulería y el odontólogo me diga -no joda quedó el equipo nuevo- un equipo de diez años veinte años y que quede nuevecito nuevamente ósea se siente uno como que... y lo va recomendando uno con el otro [...] si ve entonce eso se siente uno como todavía como útil, importante en la yo a vece me llaman hoy me llamaron y me dicen -Alfonso y que- compa hoy no voy hoy no voy -aja y qué tiene- no mi hemano estoy como todo jodón hoy me siento como enredado el mismo odontólogo me llama -no qué vente pa acávente pa acá y no tomamos una botellita de whisky vamos hacer ete trabajo- yo no ombe yo no voy por allá porque no quiero está enredado entonce como Liliam me había dicho que venía a la tres y media ¡no! voy a etá pendiente poque ajá. (AVA, HN2G2).

8)

A: ¿Cómo era su familia en ese tiempo de su infancia su infancia?

B: tengo seis el mayor tiene veintitrés año, la segunda le sigue eta embarazada tiene veinte y un año estudia aquí enfermería que ya va pa seto semestre y son jóvenes ya son jóvenes bueno no he recibido queja nadie se puede puede dar decir que los hijo de Dámaso hicieron esto o son así bueno se han potado bien hasta el momento tengo el más pequeño tiene seis año ese todavía no sabe pero todavía eta en la etapa de crecimiento pero lo demás si tengo uno de nueve año también lo tengo llevado hay inculcándole lo la buena costumbre bueno hasta el momento esa e mi familia. (JLP, HN1G1). 


\section{Categorización de los marcadores discursivos hallados en el habla de Cartagena}

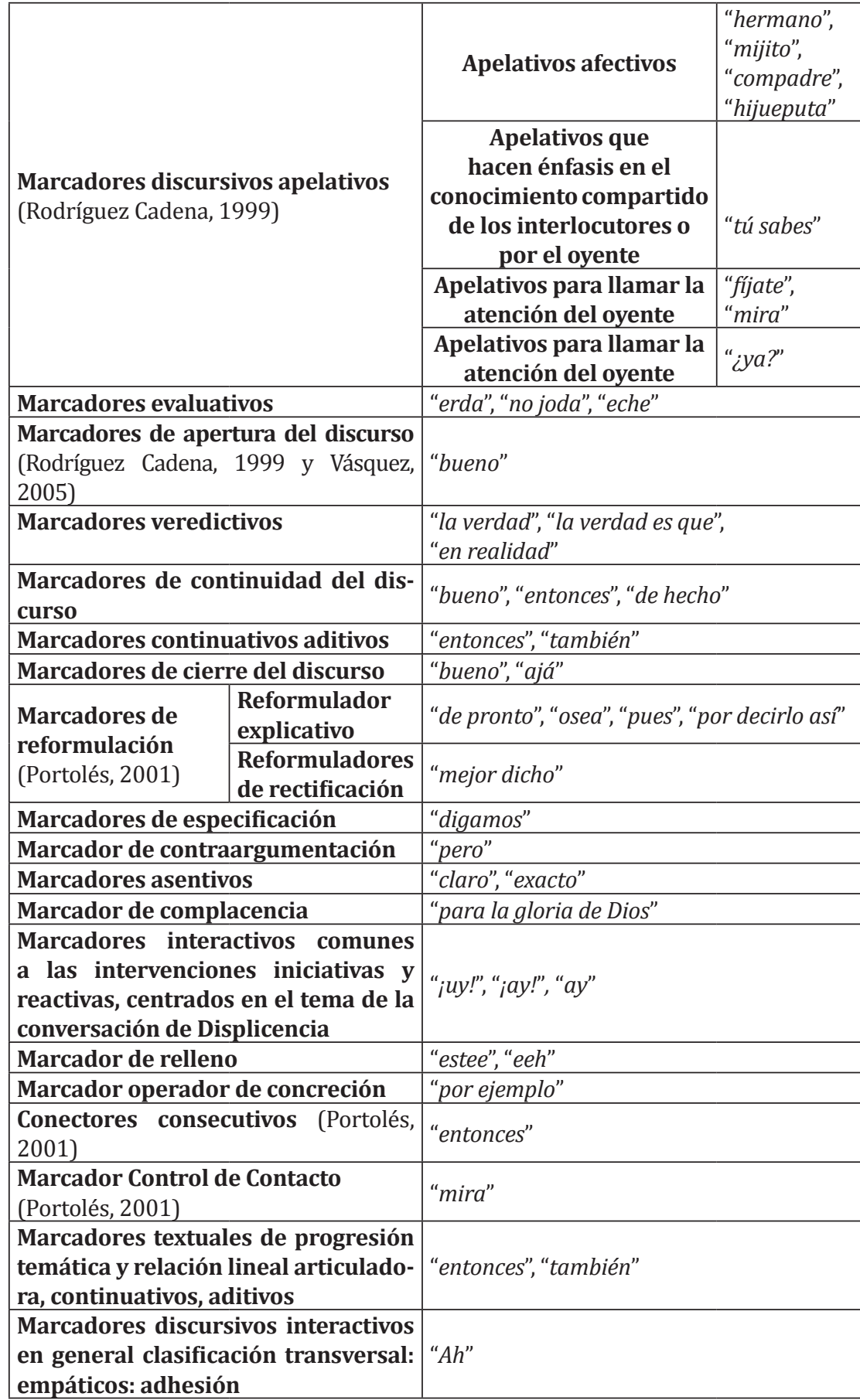




\section{Conclusiones}

Como el objetivo de esta investigación se centra en determinar, desde un enfoque sociolingüístico y discursivo, la función de los marcadores discursivos, según los géneros discursivos (narrativo, descriptivo, argumentativo, expositivo y dialogal), en el habla de Cartagena de Indias, el análisis e interpretación de los datos permite plantear las siguientes conclusiones:

Los marcadores más frecuentes en el habla de Cartagena son "entonce(s)", con una frecuencia de 799 (14, 64\%) del total de marcadores, "bueno", con 699 (12,81\%), "ya", con 563 (10,32\%), "ehh", con 530 (9,71\%), "o sea", con 467 (8,56\%), "eso", con 376 (6, 9\%), "de pronto", 271 (5\%), "pues", con 238 (4,36 \%), "asi", con 231 (4,23\%), "pero", con 223 (4,08\%), "porque", con 201 (3,68\%), “aja", con 98 (1,8\%), "la verdá(d) es que", con $61(1,18 \%)$, "ah", $51(0,93)$, "ahora" y "por ejemplo", con 48 (0,88\%), "ombe", con 43 (0,78\%), "hermano", con 39 (0,71\%), "este", con 38 (0,7\%), "digamos" y "no", con $37(0,68 \%)$, "la verdad", con $33(0,60 \%)$ y "verdad", con 27 $(0,50 \%)$. Algunos de ellos pueden presentar diversas funciones, dependiendo de la posición que ocupan en el enunciado, o de la secuencia discursiva de la que hacen parte.

De la misma manera, existe un gran número de marcadores con baja frecuencia de uso como "en ese sentido", "figúrese", "incluso", "lógicamente", "miércole", "mijito", "mire", "ñerda", "oyeron", "para la gloria de dios", "perfecto", "por decirlo asî", entre otros; con sólo un caso (1) cada uno, que requieren un seguimiento más detenido y profundo para su caracterización como propios de la ciudad de Cartagena de Indias y para su precisión funcional.

En las entrevistas semidirigidas, las mujeres son las que más producen secuencias discursivas descriptivas. De ahí que sean las informantes de este género las que más frecuencia de marcadores tienen para esta tipología (116), frente a los de sexo masculino (58). Los marcadores discursivos también están condicionados por los temas de la conversación y las diferentes fuerzas enunciativas. Así, por ejemplo, cuando se argumenta, los hablantes suelen emplear marcadores de tipo causal.

Los marcadores discursivos aparecen relacionados con factores cotextuales y sociales (sexo, edad y nivel de instrucción). Las frecuencias y los tipos de marcadores están condicionados por el nivel educativo de los informantes: los resultados arrojaron que las mujeres del nivel educativo superior y de la tercera generación 
hacían más uso de marcadores discursivos. En este caso, una informante tenía Doctorado y la otra Maestría, y ambas pertenecían a estratos sociales medio-alto. Esto permitió percibir que el discurso argumentativo utilizado por estas mujeres se estructuró con marcadores que ayudaron a la coherencia y cohesión del mismo. Los marcadores más utilizados por el género femenino fueron: "entonces", "por supuesto", "por decirlo asî", "por ejemplo", "exactamente”.

Frente a los marcadores discursivos argumentativos, los hombres (662), en general, utilizan mayor número de marcadores que las mujeres (601); sin embargo, ambos grupos siguen un patrón regular: las diferencias notables se encuentran con las mujeres de N3 de la tercera generación (91) con los hombres de su mismo rango (16).

En las secuencias expositivas las mujeres (1316) son quienes hacen mayor uso de marcadores que los hombres (866), presentándose una diferencia en más de 400 casos. Los hombres utilizan mayor número de marcadores (1001) en las secuencias narrativas que las mujeres (868). Cabe decir que las mujeres de la generación 3 del nivel 3 utilizan más marcadores que los hombres de estos mismos rasgos.

Un marcador discursivo puede cumplir funciones diferentes en el discurso, y de la misma manera puede encontrarse en diferentes tipos de texto y por personas de distinta condición social. Entre estos se pueden mencionar: "pues", "bueno", "osea".

La diferencia entre hombres y mujeres, en cuanto al uso de marcadores, resulta significativa sólo cuando se detalla la frecuencia de uso. Por ejemplo, se puede apreciar que los hombres de la primera generación tienen una frecuencia de uso de 1428 frente a 856 de las mujeres, con una diferencia de más de 500. Para la tercera generación se observa una diferencia significativa en la frecuencia de más de 500 casos. Además, a medida que las generaciones son mayores, son menos frecuentes los marcadores.

Los hablantes de ambos sexos de la primera generación y las mujeres de la tercera generación, a mayor nivel académico, usaron mayor número de marcadores. Los hombres más adultos presentan una diferencia de 292 con las mujeres de su misma generación y nivel educativo. Parece que el nivel de instrucción asociado a la profesión del informante (necesidad de usar competentemente la lengua) es un factor determinante en la elaboración discursiva, y consecuentemente, el tipo y frecuencia de marcadores discursivos. 
Según el número de marcadores por secuencia, la expositiva parece ser la más frecuente en las intervenciones de los cartageneros, seguida de la narrativa, la argumentativa, la dialogal, y finalmente, la descriptiva. Aunque hay que aclarar que, de acuerdo al formato de entrevista, la dialogal es la englobante en este caso. Esto se debe a que en el acto de la conversación los participantes hacen uso frecuente de las secuencias narrativas, descriptivas, expositivas y argumentativas en sus intervenciones.

\section{Bibliografía}

Areiza, R.; Cisnero M. \& Tabares, L. (2004). Hacia una nueva visión sociolingüística. Bogotá: Ecoe.

Becerra, S. (1985). Fonología de las consonantes implosivas en el español urbano de Cartagena de Indias. Bogotá: Instituto Caro y Cuervo.

Briz, A. (1998). El español coloquial en la conversación. Esbozo de pragmagramática. Barcelona: Ariel.

Calsamiglia, H. \& Tusón, A. (2002). Las cosas del decir. Manual del análisis del discurso. Barcelona: Ariel.

Cortés, L. (2003) ¿Qué es el análisis del discurso? Barcelona: Octaedro.

Cortés, L. (2000). "Conectores, marcadores y organizadores como elementos del discurso". En De Bustos, J.J. et. al. (Eds). I Simposio Internacional de Análisis del discurso, I. Madrid: Visor, pp. 539549.

Cortés, L. \& Camacho, M. M. (2005). Unidades de segmentación y marcadores del discurso. Elementos esenciales en el procesamiento discursivo oral. Madrid: Arco Libros.

Cueva, A. (2007). El estudio de los marcadores del discurso del Español. Desarrollo y nuevas perspectivas, León, Universidad de León (Trabajo Inédito).

Duranti, A. (1992). "La etnografía del habla: hacia una lingüística de la praxis". En Newmeyer, F. Panorama de la lingüística moderna, IV. Madrid: Visor, pp. 253-274

Galloso, M. V. (2003). El léxico disponible de Ávila, Salamanca y Zamora. Burgos- España: Fundación Instituto Castellano y Leonés de la Lengua.

Giaimo, S. (2000). Cartagena de Indias sobrellevando la crisis. Doblamiento y ciudades del Caribe colombiano. Cartagena: Observatorio del Caribe Colombiano.

González, M.(2000)."Los marcadores del discurso como piezas del engranaje textual”. En De Bustos, J.J. et. al. (Eds). I Simposio Internacional de Análisis del discurso, I. Madrid: Visor, pp. 551-564.

GIESCA. (2002). Proyecto para el estudio sociolingüístico del Español de España y América en el Caribe colombiano (PRESEEA Barranquilla). 
Hernández, J. M. \& Almeida, M. (2005). Metodología de la investigación sociolingüística. Málaga: Comares.

López, H. (1994). Métodos de investigación lingüística. Salamanca: Ediciones Colegio de España.

Martín, M.A. \& Portolés, J. (1999). “Los marcadores del discurso”. En Bosque, Ignacio \& Demonte, Violeta. (Dirs.). Gramática descriptiva de la lengua española. Madrid: Espasa-Calpe.

Martínez, M. C. (2002). Lectura y escritura de textos: perspectivas teóricas y talleres. Cali: Cátedra UNESCO para la Lectura y la Escritura.

Montolío, E. \& Unamundo, V. (2000). "El marcador del discurso "a ver" (catalán "a veure") en la interacción profesor-alumno". En En De Bustos, J.J. et. al. (Eds). I Simposio Internacional de Análisis del discurso, I. Madrid: Visor.

Moreno, F. (1990). Metodología sociolingüística. Madrid: Gredos.

Moreno, F. (1996). "Metodología del "Proyecto para el estudio sociolingüístico del Español de España y América” (PRESEEA)”, Lingüística, $\mathrm{n}^{\circ}$ 8, pp. 257-287.

Moreno, F. (1997). Trabajos de sociolingüística hispánica. Alcalá de Henares: Universidad de Alcalá.

Moreno, F. et. al. (2002). La lengua hablada en Alcalá de Henares. Corpus PREESEA. Alclál: Universidad de Alcalá.

Moya, J.A. (Coord.). (2007). El español hablado en Granada. Corpus para su estudio sociolingüístico. I Nivel de Estudios Alto. Granada: Universidad de Granada.

Moya, C. (2001). “Visión panorámica del contexto”. En Lenguaje y cognición: universos humanos. Bogotá: Instituto Caro y Cuervo-Universidad de Salamanca, vol. V.

Ochoa, L. (2008). Comunicación oral argumentativa. Estrategias didácticas. Bogotá: Magisterio.

Pérez, M. (2000). "Descripción semántico-pragmática de los conectores textuales en el nivel temático del parágrafo". En De Bustos, J.J. et. al. (Eds). I Simposio Internacional de Análisis del discurso, I. Madrid: Visor, pp. 655-668.

Poblete, M. T. (1997). "Los marcadores discursivos conversacionales en la construcción del texto oral”, Onomázein, $n^{\circ} 2$, Chile, pp. 67-81.

Poblete, M. T. (1999). "Distribución de marcadores discursivos en distintos tipos de discurso", Onomazein, ^4, Chile, pp. 53-75.

Portolés, J. (2001). Marcadores del discurso. 2a ed. Barcelona: Ariel.

PRESEEA-ALCALA I. (2002). Hablantes de instrucción superior. Alcalá de Henares: Universidad de Alcalá.

Rodríguez, Y. (1999). "Marcadores discursivos en el habla de Barranquilla", Litterae. Revista de la Asociación de Exalumnos del Seminario Andrés Bello del ICC, nº. 8, Bogotá. 
Rodríguez, Y. (1997). “Tendencias de variación sociolingüística en la Ciudad de Barranquilla", Polifonía, ${ }^{\circ} 1$, Barranquilla, Universidad del Atlántico, pp. 109-134.

Sperber, D. \& Wilson, D. (1994). La relevancia. Comunicación y procesos cognitivos. Madrid: Visor.

Schiffrin, D. (1988). "El análisis de la conversación". En Newmeyer, F. Panorama de la lingüística moderna. Cambridge: Universidad de Cambridge, pp. 299-327.

Silva-Corvalán, C. (2001). Sociolingüística y pragmática del español. Washington: Georgetown University Press.

Trudgill, P. \& Hernández, J. M. (2007). Diccionario de sociolingüística. Madrid: Gredos.

Vásquez, A. (2005). Los marcadores discursivos en la comunidad de habla de Barranquilla, Bogotá, Instituto Caro y Cuervo. Tesis de Maestría. 